\title{
Vom Unikat zum digitalen Dokument: der strukturierte radiologische Befundbericht
}

Seit Mitte 2017 gibt es die Website www.befundung.drg.de. Hier finden sich neben umfangreichen Informationen zum Thema Strukturierte Befundung auch erste Befundvorlagen, die von der AG Informationstechnologie (AGIT) gemeinsam mit anderen Arbeitsgemeinschaften in der DRG erarbeitet wurden. Prof. Dr. Thomas Hackländer, Vorsitzender der AGIT und treibende Kraft beim Thema Strukturierte Befundung, stellt die Webseite vor und beschreibt, wie er sich den „idealen“ Befundbericht der Zukunft vorstellt.

Herr Prof. Hackländer, der radiologische Befundbericht ist ein Kernelement radiologischer Tätigkeit. Wie sieht für Sie der ideale Befundbericht der Zukunft aus?

Zukünftig sollte der Befundbericht elektronisch erstellt und in einem Dateiformat gespeichert werden, das verschiedene Darstellungsformen ermöglicht: Zum einen sollte eine texturale und multimediale Visualisierung für menschliche Leser, zum anderen aber auch eine semantische Auswertung durch Computersysteme möglich sein. Auch inhaltlich sollte sich der Befundbericht weiter entwickeln: Etwa bis zur Jahrtausendwende waren viele Bilddokumente radiologischer Untersuchungen Unikate, die in Archiven der erzeugenden Radiolo- gen gelagert wurden. Aus diesem Grund lagen die Bilder dem Leser eines Befundberichtes häufig nicht vor, sodass der Bericht eine möglichst anschauliche Beschreibung der Pathologien enthalten musste. Das hat sich in der heutigen Zeit völlig gewandelt: Durch die Digitalisierung der Radiologie und der Kommunikationskanäle ist das Bilddokument jederzeit beim Leser des Befundberichtes verfügbar. Der Befundbericht kann sich deshalb auf die Bewertung der Pathologien fokussieren und die Beschreibung eher strukturiert und tabellenartig wiedergeben. Bei einer solchen Darstellungsform weiß der Leser dann genau, an welcher Stelle des Berichtes er welche Information erwarten kann.

Die DRG hat bereits 2016 eine Befundungsinitiative gestartet. Welche Motivation liegt dieser Initiative zugrunde?

Die DRG hat schon früh erkannt, dass die Erstellung des Befundberichtes nur ein Teil eines komplexeren Befundungsprozesses ist. Hierzu zählen neben der technischen Durchführung einer Untersuchung oder Intervention auch mehrere ärztliche Aufgaben: die Stellung der rechtfertigenden Indikation, die Erstellung des Befundberichtes sowie die Kommunikation mit dem Patienten und dem Zuweiser in Bezug auf den Befundbericht sowie die Kommunikation

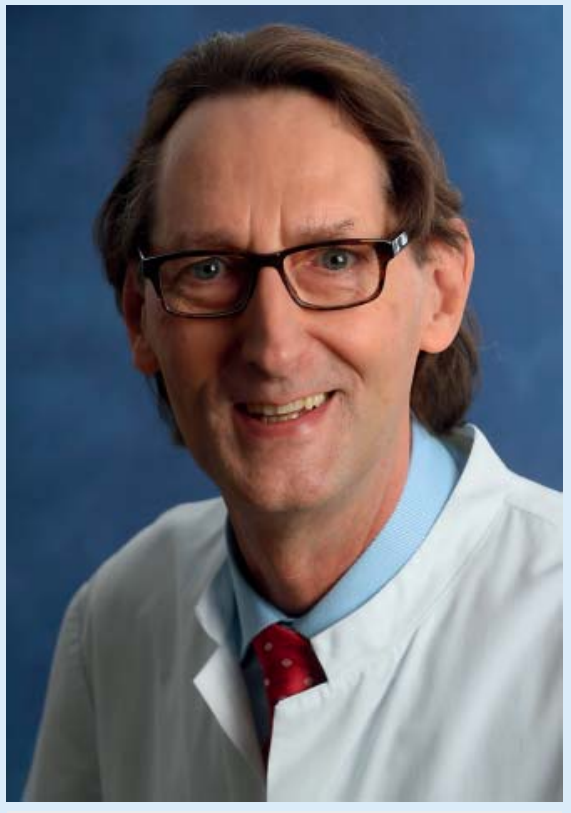

Prof. Dr. Thomas Hackländer, Vorsitzender der AG Informationstechnologie, HELIOS Klinikum Wuppertal.

im Rahmen klinischer Konferenzen. Einige dieser Prozessschritte sind zwar seit langem intuitiv gelebte Realität, müssen aber an verschieden Stellen klarer formuliert und gegeneinander abgegrenzt werden. Bei dieser Analyse wurde auch schnell deutlich, dass man die Problemfelder nicht unabhän- 
gig voneinander angehen kann, sondern dass man die Aktivitäten in einer übergreifenden Initiative bündeln sollte.

\section{Die Befundungsinitiative vereint mehrere Projekte und Ansätze. Welche sind dies im Einzelnen?}

Zentraler Punkt war die Erarbeitung einer Norm, die Begriffsdefinitionen gibt und den Prozess der Befundung in seinen Einzelschritten beschreibt. Nach mehrjähriger Abstimmung mit allen interessierten Kreisen, wie Behörden, Industrie, Anwendern und Fachverbänden wurde die Norm „DIN 25300 - 1 Prozesse in der Radiologie - Teil 1: Befundung eines bildgebenden oder bildgestützten Verfahrens" schließlich Ende 2017 veröffentlicht. Die Norm legt zwar fest, dass ein Befundbericht zu radiologischen Untersuchungen und Interventionen angefertigt werden muss, spezifiziert dessen Inhalt aber nicht. Die DRG sieht hier ergänzende Aufgaben darin, die Struktur und die inhaltlichen Rahmenbedingungen festzulegen. Eine Befundvorlage kann dabei als ein leeres Formular verstanden werden, in das die Ergebnisse einer speziellen Untersuchung eingetragen werden können. Die Vorlagen der DRG werden in einem von der IHE entwickelten Dateiformat veröffentlicht, das in erster Linie der Verteilung, nicht aber der Darstellung der Formulare dient. Das Ausfüllen der Formulare wird in der Regel in bestehenden RISoder KIS-Systemen erfolgen. Um Interessenten auch ohne Anpassung ihrer lokalen Infrastruktur einen einfachen Einstieg in die neue Technologie zu ermöglichen, stellt die DRG ein kostenloses Tool zum Ausfüllen der Vorlagen zur Verfügung. Da man bei einer Befundvorlage genau weiß, welche Information an welcher Stelle steht, kann man den texturalen Inhalt mit Codes hinterlegen. Analysieren Computerprogramme so gestaltete Befundberichte, können sie relativ einfach über die Codes den inhaltlichen Zusammenhang erfassen. Speziell zur Kodierung von Befundberichten wurde von der RSNA die RadLex-Ontologie entwickelt - leider aber nur in englischer Sprache. Um dieses Kodiersystem mit über 45000 Begriffen auch in Deutschland anwenden zu können, hat die DRG mit erheblichem personellem und finanziellem Aufwand eine Übersetzung des RadLex in die deutsche Sprache umgesetzt.

Seit Mitte 2017 gibt es mit www.befundung.drg.de eine Website, die umfangreiche Informationen zum Thema Strukturierte Befundung bietet. An wen richtet sich das Angebot?

Das Angebot richtet sich an alle Radiologen im deutschsprachigen Raum, unabhängig von der Mitgliedschaft in der Deutschen Röntgengesellschaft. Wir sind der Meinung, dass die DRG als wissenschaftliche Fachgesellschaft die Rahmenbedingungen für die medizinische Tätigkeit des Radiologen vorgeben sollte.

Die DRG bietet auf der Website auch strukturierte Befundvorlagen an. Wie werden diese entwickelt?

Die Entwicklung von Befundvorlagen erfolgt im Rahmen eines mehrstufigen Prozesses: Zunächst wird in Konsensgesprächen mit den zuweisenden klinischen Disziplinen versucht festzulegen, welche Inhalte in einem speziellen Befundbericht enthalten sein müssen. Diese Stoffsammlung wird durch eine Arbeitsgruppe der AGIT in ein funktionstüchtiges Formular überführt, das im Sinne einer „public comment"-Phase auf der Webseite der DRG veröffentlicht wird. Nach Diskussion und Einarbeitung von Änderungsvorschlägen sowie Kodierung des Inhaltes mit Begriffen aus dem RadLex-Vokabular wird die Befundvorlage dann als finales Dokument der Allgemeinheit zur Verfügung gestellt. Im Rahmen eines Qualitätssicherungsprozesses werden die Vorlagen in der Folge regelmäßig überprüft und optimiert.

Sind strukturierte Befundvorlagen nur eine praktische Hilfe für den Befundungsalltag oder auch eine implizite Aufforderung, sich den Herausforderungen der Zukunft zu stellen, sich zukunftsfest zu machen?

Sicher beides: Zum einen kann sich aus der Anwendung von Befundvorlagen eine Zeiter- sparnis dadurch ergeben, dass in den Formularen der „Normalzustand“ bereits als Voreinstellung eingetragen werden kann, sodass der Radiologe nur noch die Pathologien eintragen muss. Eine andere Hilfe besteht in der Unterstützung junger Kollegen in der Ausbildung, für die die Befundvorlagen ein „How to“ ihrer klinischen Praxis sein können. Andererseits werden KI-Systeme innerhalb weniger Jahre Einzug in die medizinische Versorgung halten. Um diese Technologien für die Patienten gewinnbringend einsetzen zu können, müssen auch die radiologischen Untersuchungsergebnisse inhaltlich durch Computer auswertbar sein. Für diese Anwendung kommt man kaum an strukturierten Befundberichten vorbei.

\section{Abschließend noch eine Prognose zu Ihrem idealen Befundbericht der Zukunft. Wann wird dieser Ihrer Meinung nach gelebte Wirklichkeit sein?}

Da die technischen und organisatorischen Rahmenbedingungen aufgebaut sind, könnten die Befundberichte schon heute in der moderneren Form verfasst werden. Allerdings wird es noch einige Jahre dauern, bis sich die strukturierte Befundung auf breiter Front durchsetzen wird. Dies ist im Wesentlichen zwei Dingen geschuldet: Zum einen müssen sich sowohl die Radiologen als auch die Zuweiser an die neue Form gewöhnen, wobei die Akzeptanz auf beiden Seiten sehr hoch ist, sobald man den Schritt einmal gegangen ist. Der zweite Punkt ist schwieriger umzusetzen: Die Inhalte der Befundvorlagen müssen erstellt werden. Das ist in jedem Einzelfall ein zeitaufwendiger und häufig mühseliger Weg. Es wäre sicher hilfreich, wenn die Radiologen, die die Befundvorlagen erarbeiten, eine ähnliche Wertschätzung erhalten würden, wie für die Publikation einer wissenschaftlichen Arbeit.

\section{WEITERE INFORMATIONEN \\ www.befundung.drg.de \\ www.agit.drg.de}

